\title{
RECUPERAÇÃO DE COBRE ATRAVÉS DE ELETRO- OBTENÇÃO DE UMA SOLUÇÃO EXTRAÍDA SUPERCRITICAMENTE DE PLACAS DE CIRCUITO IMPRESSO
}

\author{
M. D. C. R. da SILVA ${ }^{1}$, C. O. CALGARO ${ }^{1}$, D. F. SCHLEMMER ${ }^{1}$, E. V. MAZIERO ${ }^{1}$, E. H. \\ TANABE $^{1}$ e D. A. BERTUOL ${ }^{1}$ \\ ${ }^{1}$ Universidade Federal de Santa Maria, Departamento de Engenharia Química \\ E-mail para contato: m.dalcost@hotmail.com
}

\begin{abstract}
RESUMO - As placas de circuito impresso (PCI's) são componentes fundamentais dos equipamentos elétricos e eletrônicos. Dentre os metais presentes nas PCI's, o cobre está contido em maior percentual. O objetivo deste estudo foi recuperar o cobre na forma de depósito metálico a partir de uma solução de cobre extraída sob condições supercríticas com $\mathrm{CO}_{2}$ na presença de cosolventes $\left(\mathrm{H}_{2} \mathrm{O}_{2} \mathrm{e}\right.$ $\mathrm{H}_{2} \mathrm{SO}_{4}$ ). Ensaios com uma solução sintética de $\mathrm{CuSO}_{4}$ foram realizados para determinar a densidade de corrente ótima no intervalo de 250 a $540 \mathrm{~A} / \mathrm{m}^{2}$. Na densidade de corrente determinada foi realizada a eletro-obtenção da solução real. Utilizando a densidade de corrente de $250 \mathrm{~A} / \mathrm{m}^{2}$, atingiu-se então uma eficiência de corrente em torno de $100 \%$ para a solução real. O depósito obtido dessa eletroobtenção com a solução real apresentou $95,97 \%$ de cobre, indicando que a recuperação do cobre pode ser realizada por esse método utilizando soluções extraídas supercriticamente das PCI's.
\end{abstract}

\section{INTRODUÇÃO}

O desenvolvimento tecnológico propicia a rápida substituição dos equipamentos elétricos e eletrônicos, o que torna-se um problema de ordem global. As placas de circuito impresso (PCI's) são componentes fundamentais dos equipamentos eletroeletrônicos (EEE) e sua reciclagem é motivada pela quantidade significativa de metais presentes, além do apelo ambiental (Veit et al., 2006). De acordo com Huang et al.(2009), a pureza dos metais contidos nas PCI's descartadas é superior a 10 vezes a quantidade contida nos minerais. Dentre os metais que estão presentes nas PCI's, o cobre aparece em maior quantidade (Huang et al., 2009). Além do alto grau de pureza, o cobre ainda apresenta um valor significativo no mercado de comodites (Guimarães et al., 2014).

O tratamento adequado das placas de circuito impresso além de vantajoso se faz necessário, principalmente, pela quantidade de substâncias tóxicas presentes em sua composição, como os retardantes de chama, plásticos e metais pesados, que podem causar sérios problemas ambientais e até mesmo danos à saúde humana se não dispostas corretamente (Huang et al., 2009). Devido a isso, vêm sido estudados métodos de reciclagem desses materiais utilizando processamento mecânico, térmico e químico, ou a combinação desses (Park et al,2009).Os fluidos supercríticos também vêm sendo considerados como uma 
alternativa aos métodos convencionais, devido as suas propriedades vantajosas de transferência de massa em relação aos demais solventes (Herrero et al., 2010).

A eletrometalurgia é um dos métodos empregados na recuperação de metais de PCI's, a partir do qual o metal que se encontra contido em solução, geralmente oriunda de hidrometalurgia, é recuperado em sua forma metálica pelo uso de energia. A eletro-obtenção é o estágio final de extração de cobre, zinco, níquel e de muitos outros metais não ferrosos de seu minério (Shakarji et al., 2013). Nesse contexto, o presente trabalho teve como objetivo a recuperação de cobre na forma de depósitos metálicos de uma solução oriunda de extração com $\mathrm{CO}_{2}$ supercrítico e cosolventes $\left(\mathrm{H}_{2} \mathrm{SO}_{4} \mathrm{e} \mathrm{H}_{2} \mathrm{O}_{2}\right)$ pelo emprego de eletro-obtenção.

\section{METODOLOGIA}

Ensaios de eletro-obtenção foram utilizados para avaliar o comportamento da obtenção do cobre na forma de depósitos metálicos a partir da solução extraída supercriticamente com $\mathrm{CO}_{2}$ e cosolventes $\left(\mathrm{H}_{2} \mathrm{SO}_{4}\right.$ e $\left.\mathrm{H}_{2} \mathrm{O}_{2}\right)$ e, verificar a possibilidade de obter o metal separado dos demais por esse método, depois da extração supercrítica. Primeiramente utilizou-se uma solução sintética de $\mathrm{CuSO}_{4}$, com concentração próxima a $15 \mathrm{~g} / \mathrm{L}$, para determinar a densidade de corrente ótima, dentre as seguintes: $250,370,455$ e $540 \mathrm{~A} / \mathrm{m}^{2}$, de acordo a eficiência de corrente resultante. A concentração de $15 \mathrm{~g} / \mathrm{L}$ foi utilizada pois a concentração máxima obtida nos experimentos de extração supercrítica das PCIs foi de $15,46 \mathrm{~g} / \mathrm{L}$, nas condições: razão sólido:líquido $1: 20, \mathrm{H}_{2} \mathrm{SO}_{4}(2,5 \mathrm{M})$ e $20 \% \mathrm{H}_{2} \mathrm{O}_{2}$, durante 20 min. de extração a $35^{\circ} \mathrm{C}$ e 7,5 MPa.

A célula de eletro-obtenção utilizada nos ensaios está apresentada na Figura 1. Essa célula é composta por dois compartimentos separados por uma membrana aniônica (PCacid 60/PCA Ion Exchange Membranes $\mathrm{GmbH}$ ) com área útil de $25 \mathrm{~cm}^{2}$. A corrente foi fornecida à célula por uma fonte (PS-7000, iCEL Manaus). Um ânodo de platina/irídio coberto de titânio com área em torno de $10 \mathrm{~cm}^{2}$ foi inserido no compartimento anódico, por onde circulou a solução anódica de $\mathrm{Na}_{2} \mathrm{SO}_{4}(1 \mathrm{M})$. Já o cátodo, que foi constituído de aço inox polido, com área de aproximadamente $9 \mathrm{~cm}^{2}$, foi colocado, durante cada ensaio, no compartimento catódico, por onde circulou a solução catódica. As soluções catódicas utilizadas dividiram-se em: sintética $\left(\mathrm{CuSO}_{4}\right)$ e real (obtida por extração supercrítica). As soluções foram recirculadas nos dois compartimentos a partir de bombas centrífugas, no catódico a vazão de circulação foi de aproximadamente $2,2 \mathrm{~L} / \mathrm{min}$, já no compartimento anódico a vazão foi em torno de 1,5 $\mathrm{L} / \mathrm{min}$. Os ensaios foram realizados com $\mathrm{pH}$ inicial de $4 \mathrm{em}$ ambas as soluções, monitoradas com pHmetro e ajustadas no decorrer do experimento com $\mathrm{NaOH}(1 \mathrm{M}, 2 \mathrm{M}$ e $4 \mathrm{M})$ e $\mathrm{H}_{2} \mathrm{SO}_{4}$ (4M) à temperatura ambiente. Os ensaios para a escolha da densidade de corrente foram realizados no tempo de $2 \mathrm{~h}$. Depois de determinada a densidade de corrente ideal os ensaios foram realizados durante $5 \mathrm{~h}$.

$\mathrm{O}$ cátodo foi pesado antes e depois de cada ensaio. A área útil do cátodo também foi estimada antes de cada experimento. A concentração das soluções catódicas foi determinada por Espectrometria de Absorção Atômica de Chama (F-AAS). E, a eficiência de corrente foi determinada a partir da equação de Faraday (Equação 1) e da massa de depósito eletro-obtido, utilizando a Equação 2 (Schlesinger e Paunovic, 2000). 


$$
m_{\text {teórico }}=\frac{M_{\text {cobre }} \times i \times t}{n \times F}
$$

Eficiência de corrente $(\%)=\frac{m_{\text {depósito }} \times 100}{m_{\text {teórico }}}$

Depois de determinada a corrente ideal, esta foi utilizada uma eletro-obtenção de $5 \mathrm{~h}$ para avaliar o comportamento da eficiência de corrente de acordo com a redução da concentração de cobre em solução, determinada por F-AAS.

Figura 1 - Esquema da célula de eletroobtenção com dois compartimentos.

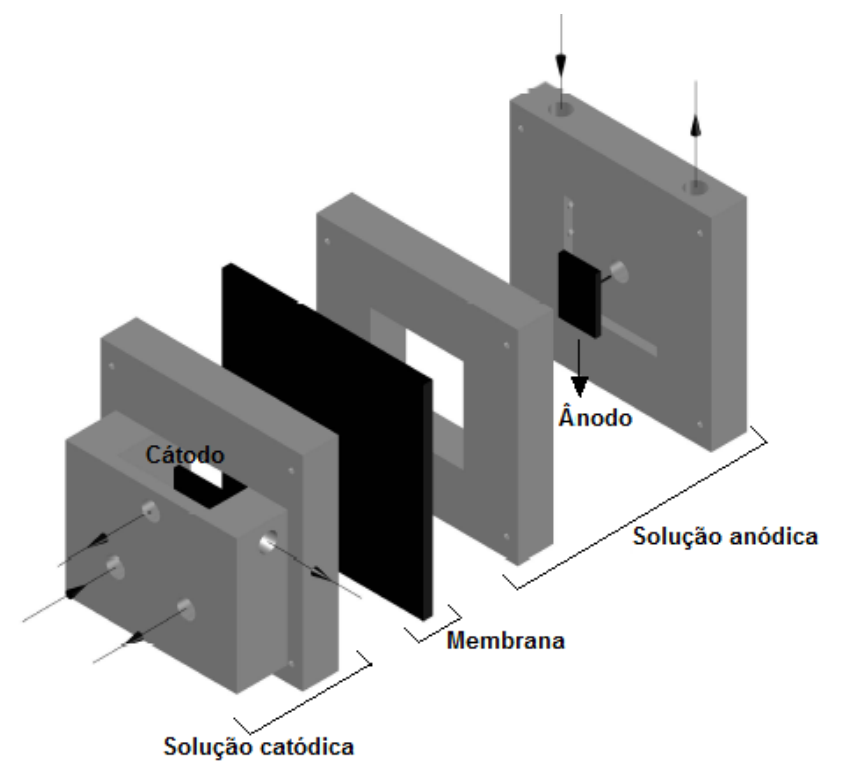

Adicionou-se $\mathrm{NaOH}(2 \mathrm{M}$ e $4 \mathrm{M})$ à solução real até que o $\mathrm{pH}$ fosse igual a 4 e a concentração de cobre foi então determinada por F-AAS. Quando o $\mathrm{pH}=4$ foi alcançado, realizou-se uma filtração para remoção do precipitado formado. O cobre foi eletro-obtido a partir dessa solução real durante $5 \mathrm{~h}$ e, a eficiência de corrente foi calculada em relação a massa de depósito e em relação a massa de cobre que foi removida da solução, determinada por F-AAS. O depósito metálico foi caracterizado por meio da dissolução de uma pequena fração do depósito em ácido nítrico a $60^{\circ} \mathrm{C}$, durante $1 \mathrm{~h}$, sob agitação de $600 \mathrm{rpm}$. O cobre presente na solução resultante foi determinado por F-AAS.

\section{RESULTADOS E DISCUSSÃO}

\subsection{Eletro-obtenção do cobre com a solução sintética}

Para determinar a densidade de corrente ótima de acordo com a eficiência de corrente, os depósitos de cobre foram obtidos a partir de soluções sintéticas de $\mathrm{CuSO}_{4}$, com concentração média de $15 \mathrm{~g} / \mathrm{L}$ de cobre, em 4 densidades de corrente diferentes. A Figura 2 apresenta as eficiências de corrente nas densidades de corrente de 250,370 e $455 \mathrm{~A} / \mathrm{m}^{2}$. A maior eficiência de corrente $(99,3 \%)$ foi alcançada quando a densidade de corrente foi de 250 $\mathrm{A} / \mathrm{m}^{2}$ foi aplicada. Logo, a densidade de corrente de $250 \mathrm{~A} / \mathrm{m}^{2}$ foi utilizada nos estudos sequentes. 
Figura 2: Determinação da densidade de corrente ideal, nas condições: $\mathrm{pH}=4$, temperatura ambiente, durante $2 \mathrm{~h}$, a partir de solução sintética de $\mathrm{CuSO} 4$, à temperatura ambiente.

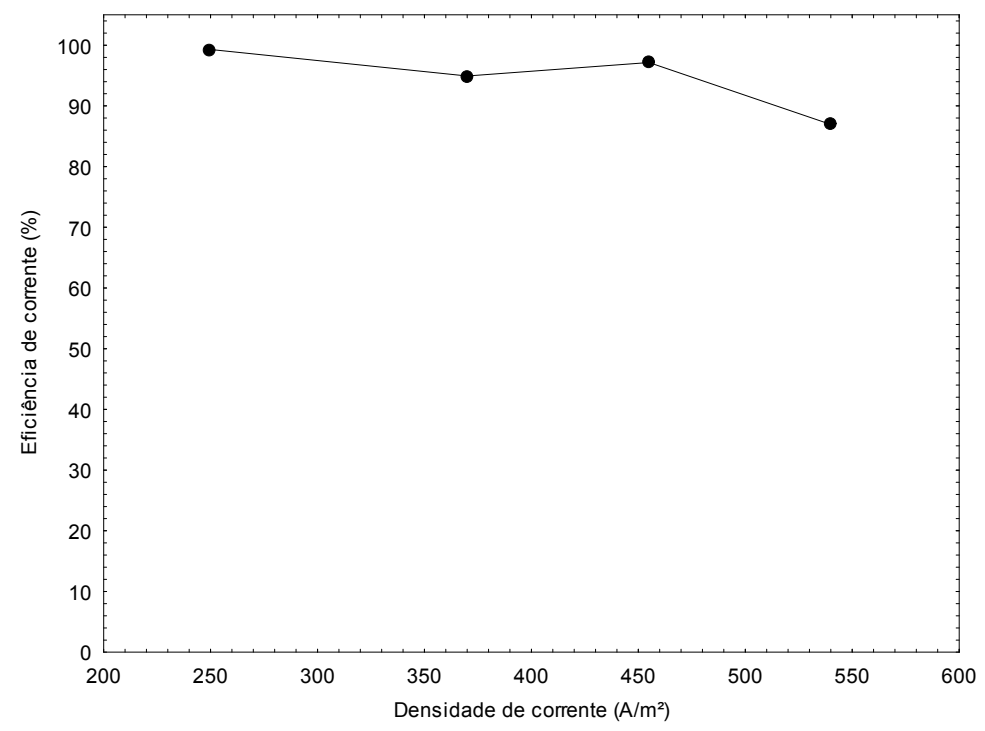

Determinada a densidade de corrente ótima $\left(250 \mathrm{~A} / \mathrm{m}^{2}\right)$, foi realizada outra eletroobtenção de $5 \mathrm{~h}$ a partir da solução sintética para avaliar a redução progressiva da concentração de cobre na solução ao longo do tempo. A Figura 3 mostra o comportamento da concentração em função do tempo e a eficiência de corrente em cada ensaio. Pode-se observar que na Figura 3 que a eficiência de corrente no ensaio foi de $98 \%$.

Figura 3: Variação da concentração de acordo com o tempo de eletro-obtenção, com a densidade de corrente de $250 \mathrm{~A} / \mathrm{m}^{2}, \mathrm{pH}=4$, solução sintética de $\mathrm{CuSO} 4$, à temperatura ambiente.

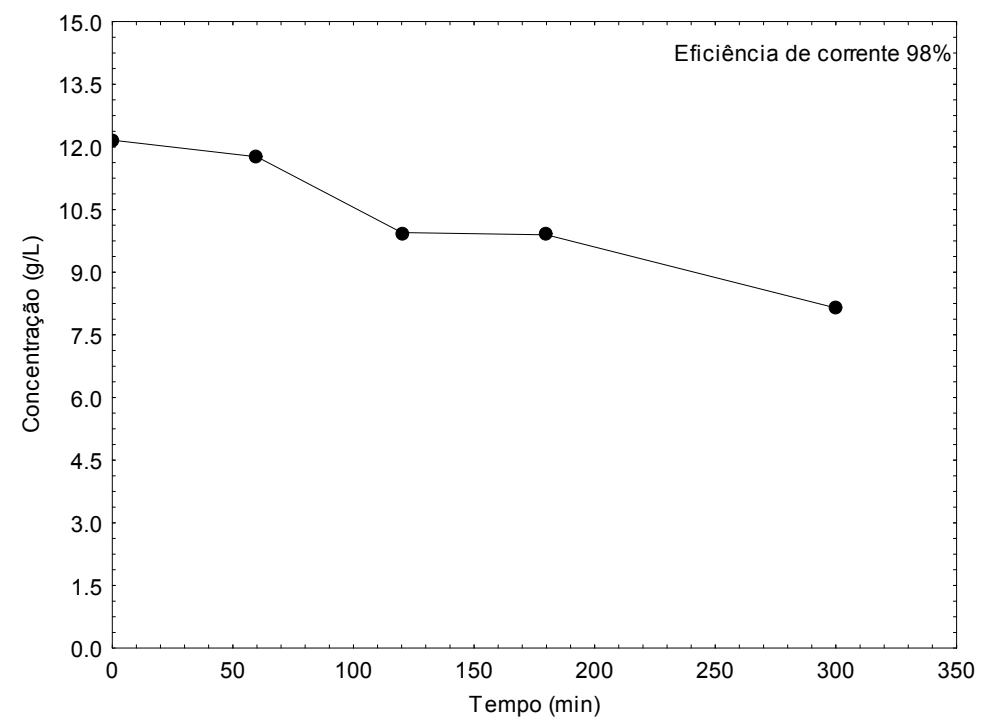




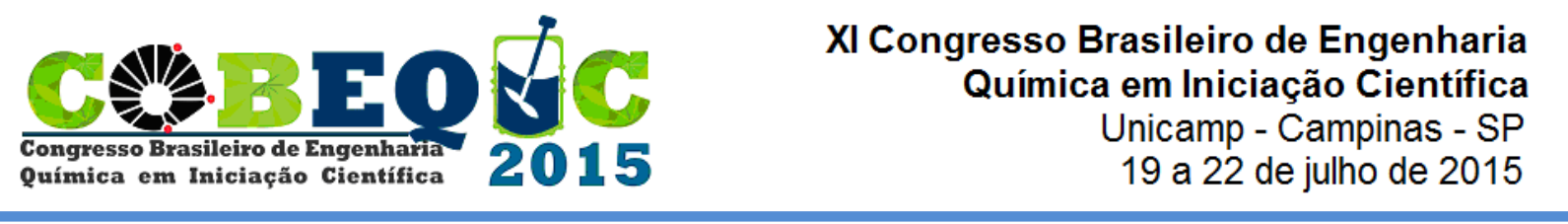

\subsection{Eletro-obtenção do cobre a partir da solução real}

Utilizando a densidade de corrente ótima para a solução sintética, o ensaio de eletroobtenção de cobre com a solução real foi realizado durante $5 \mathrm{~h}$. Na Figura 4 pode-se observar o comportamento da concentração de cobre na solução real em função do tempo de ensaio. A eficiência de corrente obtida a partir de F-AAS foi de 99,33\%. Observou-se que a eficiência de corrente manteve um comportamento semelhante ao previsto pelos ensaios com a solução sintética.

A partir da Figura 4 pode-se observar que a concentração inicial de cobre da solução real no início do ensaio foi de $5,11 \mathrm{~g} / \mathrm{L}$, inferior aos $15 \mathrm{~g} / \mathrm{L}$ obtidos por meio da extração supercrítica. Essa redução na concentração deve-se à adição das soluções de $\mathrm{NaOH}(2 \mathrm{M}$ e 4M) para corrigir o $\mathrm{pH}$ para o valor de 4 .

Figura 4: Variação da concentração do $\mathrm{Cu}$ na solução real de acordo com o tempo de eletro-obtenção, utilizando uma densidade de corrente de $250 \mathrm{~A} / \mathrm{m}^{2}, \mathrm{pH}=4$, à temperatura ambiente.

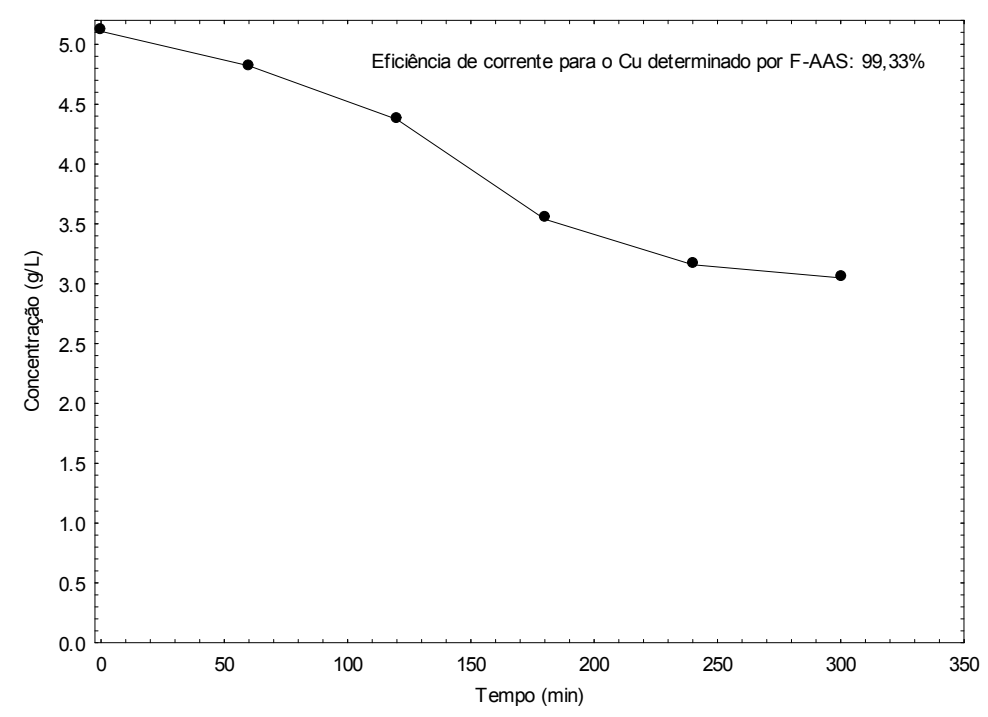

\section{CONCLUSÃO}

Ensaios com a solução sintética de $\mathrm{CuSO}_{4}$ mostraram que a densidade de corrente 250 $\mathrm{A} / \mathrm{m}^{2}$ levou a maior eficiência de corrente para a deposição de cobre, dentre as densidades de correntes testadas $\left(250,370,455\right.$ e $\left.540 \mathrm{~A} / \mathrm{m}^{2}\right)$. Ensaios com a solução sintética sob as condições ótimas durante $5 \mathrm{~h}$ mostraram que a eficiência de corrente não se altera apreciavelmente. Ensaios com a solução real na densidade de corrente $250 \mathrm{~A} / \mathrm{m}^{2}$ durante $5 \mathrm{~h}$ mostraram que a eficiência de corrente obtida foi próxima a $100 \%$ e o depósito obtido foi constituído por $95,97 \%$ de cobre. Portanto, pode-se inferir que é possível eletro-obter o cobre a partir de soluções extraídas supercriticamente de PCIs, obtendo-se um depósito com alta pureza.

\section{REFERÊNCIAS}


HUANG, K.; GUO, J.; XU, Z. Review - Recycling of waste printed circuit boards: A review of current technologies and treatment status in China. J. Hazard. Mater., v.164, p. 399408, 2009.

GUIMARÃES, Yuri F.; SANTOS, Iranildes D.; DUTRA, Achilles J.B.; Direct recovery of copper from printed circuit boards (PCBs) powder concentrate by a simultaneous electroleaching-electrodeposition process. Hydrometallurgy, 149 (2014) 63-70, 2014.

PARK, Y.J.; FRAY, D.J. Recovery of high purity precious metals from printed circuit boards. J. Hazard. Mater., v. 164, p. 1152-1158, 2009.

SHAKARJI, R. A.; HE, Y.; GREGORY, S. Performance evaluation of acid mist reduction techniques in copper electrowinning. Hydrometallurgy, v. 131-132, p. 76-80, 2013.

VEIT, H. M.; BERNARDES, A. M.; FERREIRA, J. Z.; TENÓRIO, J. A. S.; MALFATTI, C. F.Recovery of copper from printed circuit boards scraps by mechanical processing and electrometallurgy. J. Hazard. Mater., v. B137, p.1704-1709, 2006. 